\title{
Salting-out Assisted Liquid-Liquid Extraction for the Determination of Multiresidue Pesticides in Alcoholic Beverages by High Performance Liquid Chromatography
}

\author{
Tesfa Bedassa $^{1,2}$, Negussie Megersa ${ }^{1}$, Abera Gure ${ }^{1,3,}$, \\ ${ }^{1}$ Department of Chemistry, Addis Ababa University, Addis Ababa, Ethiopia \\ ${ }^{2}$ Department of Chemistry, Wollega University, Nekemte, Ethiopia \\ ${ }^{3}$ Department of Chemistry, Jimma University, Jimma, Ethiopia
}

Email address:

aberagure@gmail.com (A. Gure), gureabera@ayahoo.com (A. Gure)

*Corresponding author

\section{To cite this article:}

Tesfa Bedassa, Negussie Megersa, Abera Gure. Salting-out Assisted Liquid-Liquid Extraction for the Determination of Multiresidue Pesticides in Alcoholic Beverages by High Performance Liquid Chromatography. Science Journal of Analytical Chemistry.

Vol. 5, No. 3, 2017, pp. 38-45. doi: 10.11648/j.sjac.20170503.11

Received: May 1, 2017; Accepted: May 8, 2017; Published: July 4, 2017

\begin{abstract}
A salting-out assisted liquid-liquid extraction (SALLE) method followed by high-performance liquid chromatography with ultraviolet-visible detector (HPLC-UV/Vis) has been proposed for determination of five multiclass pesticides residues including, atrazine, ametryn, terbutryn, carbaryl and chlorothalonil from various alcoholic beverages: beer, wine and Ethiopian honey wine $(T e j)$. Experimental parameters influencing the extraction efficiency of the method such as the type and concentration of salt, volume of acetonitrile (the extraction solvent) and $\mathrm{pH}$ of the sample were assayed and the optimum conditions were established. Under the optimum experimental conditions, matrix-matched calibration curves were constructed using beer sample as the representative matrix and good linearity over wide concentration ranges were obtained with coefficient of determination $\left(\mathrm{r}^{2}\right)$ of 0.997 or better. The limits of detection (LOD) and quantification (LOQ) of the method, which were determined as 3 and 10 times a signal-to-noise ratio $(\mathrm{S} / \mathrm{N})$, were in the ranges of 1.3-3.9 and 4.5-12.8 $\mu \mathrm{g} \mathrm{L}^{-1}$, respectively. Precisions studied in terms of repeatability and intermediate precision (with-in lab reproducibility) at two concentration levels have demonstrated acceptable \%RSD values, which were less than $10 \%$ in both cases. The applicability of the method was also investigated by analyzing various alcoholic beverages and demonstrated satisfactory recoveries in the ranges of $71-104 \%$ with their corresponding \%RSDs less than $10 \%$ in all cases. The results of the study revealed that the developed SALLE method is selective and efficient sample preparation procedure prior to quantitative analysis of the target analytes by HPLC-UV/Vis.
\end{abstract}

Keywords: Salting-Out Liquid-Liquid Extraction, Multiclass Pesticides, Alcoholic Beverages, High Performance Liquid Chromatography, Ultraviolet-Visible Detector

\section{Introduction}

These days, various pesticides including herbicides, insecticides and fungicides are used to control and/or destroy pests that may affect crop yield. Nevertheless, their wide use in or on agricultural products might be resulted in the occurrence of residues of these chemicals and their metabolites in raw as well as processed food commodities, water and soil [1]. Alcoholic beverages which are usually prepared from various agricultural products and are considered as a part of human diet [2] could also be directly or indirectly contaminated by these pesticides and subsequently, could affect the health of consumers [3-9]. To monitor and control the health of consumers, legislative authorities and monitoring organizations of different countries, have set maximum residue limits (MRLs) of pesticide residues in various raw and processed foods, including alcoholic beverages [9-12]. Therefore, determination of the residual levels of pesticides in various 
alcoholic beverages is crucial to ensure their safety consumption. In this regard, it is imperative to develop simple, rapid, selective and environmentally benign analytical methods for precise determination of trace level multiclass pesticide residues in different alcoholic beverages in order to monitor and control the use of pesticides.

Various analytical techniques have been reported for trace level determination of pesticide multiresidues in alcoholic beverages. Gas chromatography (GC) with various detectors including flame ionization detector (FID) $[13,14]$, electron capture (ECD) [15], nitrogen-phosphorus detection (NPD) $[15,16]$, mass spectrometric detector (MS) $[7,8,17]$, tandem mass spectrometry (MS/MS) [5], capillary liquid chromatography coupled with diode array detection (cHPLCDAD) [3], ultra-high pressure liquid chromatography coupled to MS/MS (UHPLC-MS/MS) [4] and liquid chromatography with MS/MS (LC-MS/MS) [18] have been used for determination of pesticides residues in wine. UHPLC-MS/MS [4], GC-ECD, GC-MS [19] and LC-MS/MS [20] were reported for determination of pesticide residues in beer [20]. HPLC-DAD [9] was reported for determination of pesticide residues in Ethiopian honey wine, locally called Tej, which is one of the national known home brewed Ethiopian alcoholic beverages and it is prepared from fermented honey and special kind of hops called Gesho (Rhamnus prinoides) [2, 9, 21, 22]. LC-MS/MS [6] was also reported for analysis of pesticide residues in honey liqueur.

Various sample preparation methods were reported for the selective extraction and preconcentration of pesticides residues, prior to their quantitative determination. In spite of its numerous disadvantages, such as time consuming, labor intensiveness, use of large sample size and high volumes of expensive and hazardous organic solvents, solid-phase extraction (SPE) [7, 8, 17, 18] has been widely used for extraction and/or preconcentration of pesticide residues from alcoholic beverages. Solid-phase microextraction (SPME) [5] has also been reported for extraction of pesticides from wine. However, SPME is expensive, uses fragile and easily broken fibres, has limited lifetime and sometimes sample carry-over could be a problem [23]. Other sample preparation methods such as supported liquid membrane (SLM) [9], microporous membrane liquid-liquid extraction (MMLLE) [13, 14], hollow-fibre liquid phase microextraction (HF-LPME) [4], ultrasound-vortex-assisted dispersive liquid-liquid microextraction (USVA-DLLME) [16], dispersive liquidliquid microextraction (DLLME) [6] and quick, easy, cheap, effective, rugged and safe (QuEChERS) [6] as well as dispersive solid-phase extraction (d-SPE) [17] have also been reported for analysis of pesticide multiresidues in alcoholic beverages. Though, these procedures are relatively simple, rapid, cheap and attractive alternative for sample preparation, they are still demanding special equipment, reagents and/or toxic organic solvents.

Recently, salting-out assisted liquid-liquid extraction (SALLE) has gained particular recognition for extraction and/or pre-concentration of different organic compounds. SALLE is a variation of solvent extraction that uses water- miscible organic solvents such as acetone, acetonitrile, ethylacetate and isopropanol $[26,30,36]$. In the procedure, addition of appropriate amount of salts such as magnesium sulfate $\left(\mathrm{MgSO}_{4}\right)$, ammonium sulfate $\left(\left(\mathrm{NH}_{4}\right)_{2} \mathrm{SO}_{4}\right)$, sodium chloride $(\mathrm{NaCl})$, calcium chloride $\left(\mathrm{CaCl}_{2}\right)$, potassium carbonate $\left(\mathrm{K}_{2} \mathrm{CO}_{3}\right)$ and calcium sulfate $\left(\mathrm{CaSO}_{4}\right)$ reduces the mutual miscibility of the two liquids, i.e., the aqueous sample and water-miscible organic solvent, causing formation of a two-phase system with the simultaneous extraction of the target analytes into the organic phase [36]. The method is simple, fast, cheap and environmentally friendly-and its extract is also compatible with HPLC/LC, CE and/or GC instruments [36, 37]. SALLE has been applied for extraction of various organic compounds including $\alpha$-dicarbonyl compounds in beer [24] and wines [25]; biogenic amines in fruit juices and alcoholic beverages [26]; N-nitrosamines in skin care cosmetics [27]; trimetazidine in rat plasma [28]; methoxetamine in rat tissues (rat brain, liver and lungs) [29]; sulfonamides from different matrices (tea, water, milk, honey, human urine, plasma and blood) [30, 31]; temozolomide from plasma [32]; vitamin $\mathrm{K}$ homologues in human plasma [33]; multi-mycotoxin biomarkers in pig urine [34]; betalactam antibiotics in infant dairy products [35]; benzimidazole fungicides [36]; sulfonylurea herbicides in water and banana juice samples [37]; triazole pesticides in water samples [38]; multiresidue pesticides in surface, ground and drinking water [39]; and chlorophenols in wine [40]. However, to date there is no report on the use of SALLE procedure in combination with HPLC-UV/Vis for analysis of multiclass pesticide residues in alcoholic beverages.

Therefore, in this work, the SALLE method combined with HPLC-UV has been proposed for selective extraction and preconcentration as well as quantitative determination of five multiclass pesticides; namely, carbaryl, atrazine, ametryn, terbutryn and chlorothalonil from alcoholic beverages including wine, beer and Ethiopian honey wine $(T e j)$. Various parameters affecting the extraction efficiency of the technique were investigated so as to establish the optimum conditions for extensive future applications.

\section{Materials and Methods}

\subsection{Chemicals and Reagents}

All chemicals and reagents used in this study were of analytical grade while the solvents were of HPLC grade. Acetonitrile from Ashland Industries Italia S.R.L. (Busnago, Lombardy, Italy) and ultrapure water, obtained after purification with double distiller A8000 Aquatron water still (Bibby Scientific Ltd, Staffordshire, United Kingdom) were used as a mobile phase. Sodium carbonate $\left(\mathrm{Na}_{2} \mathrm{CO}_{3}\right)$ and $\mathrm{MgSO}_{4}$ were purchased from Fisher Scientific Company L. C. C. (Pittsburgh, USA); $\mathrm{NaCl},\left(\left(\mathrm{NH}_{4}\right)_{2} \mathrm{SO}_{4}\right.$ and hydrated sodium hydrogen phosphate $\left(\mathrm{NaH}_{2} \mathrm{PO}_{4} \cdot 2 \mathrm{H}_{2} \mathrm{O}\right)$ obtained from BDH Laboratory supplies (Poole, England) were used during the experimental studies. 
Analytical pesticide standards of carbaryl and chlorothalonil were purchased from Sigma Aldrich (St. Louis, MO, USA), whereas atrazine, ametryn and terbutryn were obtained from Dr. Ehrenstorfer (Augusburg, Germany). The chemical structures, common names, abbreviations and the $\mathrm{pk}_{\mathrm{a}}$ of the target pesticides are given in Figure 1. Stock standard solutions containing $1000 \mathrm{mg} \mathrm{L}^{-1}$ of each pesticide were prepared in acetonitrile and stored in dark at $4^{\circ} \mathrm{C}$.
Intermediate working solution containing $20 \mathrm{mg} \mathrm{L}^{-1}$ of each standard was also prepared in acetonitrile. Working standard solutions were prepared daily from a mixed intermediate standard solution by diluting with deionized water and then used for optimization of the parameters affecting the SALLE procedure as well as method validation. All solutions were stored under refrigeration below $4{ }^{\circ} \mathrm{C}$ when not in use.<smiles>CNC(=O)Oc1cccc2ccccc12</smiles><smiles>CCNc1nc(Cl)nc(NC(C)C)n1</smiles><smiles>CCNc1nc(NC(C)C)nc(SC)n1</smiles>

Carbaryl: $\mathrm{pK}_{\mathrm{a}} 10.4 ; \log$ P 2.36

Atrazine: $\mathrm{pK}_{\mathrm{a}} 1.7 ; \log \mathrm{P} 2.7$

Ametryn: $\mathrm{pK}_{\mathrm{a}} 10.07 ; \log \mathrm{P} 2.63$

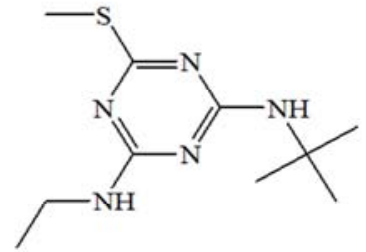

Terbutryn: $\mathrm{pK}_{\mathrm{a}} 4.3 ; \log \mathrm{P} 3.7$

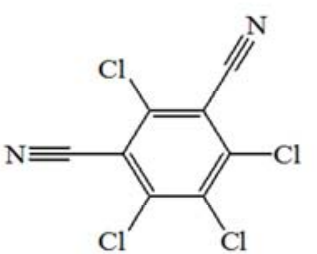

Chlorothalonil: $\log$ P 2.94

Figure 1. The chemical structures, common names, abbreviations and octanol-water partition coefficients (log $P$; at pH 7 and $\left.20^{\circ} \mathrm{C}\right)$ and $p K_{a}$ of the target pesticides.

\subsection{Instruments and Equipment}

Chromatographic analyses were performed using Agilent Technologies 1200 infinity series HPLC, equipped with quaternary pump, vacuum degasser, autosampler and UV/Vis detector all purchased from Agilent Technologies (Waldbronn, Germany). Chromatographic separation was carried out using Eclipse plus $\mathrm{C}_{18}$ column (100 x $4.6 \mathrm{~mm} \mathrm{I}$. D., $\quad 3.5 \mu \mathrm{m}$ particle sizes) obtained from Agilent Technologies. Data acquisition and processing were accomplished with LC Chemstation software, (B.02, 01-R1).

A pH meter, Adwa, model 1020 (Romania, Europe) was used for measurement of $\mathrm{pH}$. An ultrasonic heater, Dacon ${ }^{\circledR}$, Dacon laboratories Ltd (St. Hove, East Sussex), centrifuge, Model 800 (China, Beijing) and 15-mL centrifuge tube, corning incorporated, (Corning, NY, Mexico) were used during sample preparation.

\subsection{Chromatographic Conditions}

Chromatographic separations were carried out based on the earlier reported findings [41]. An isocratic elution comprising of $45 \%$ ultrapure water (solvent A) and 55\% acetonitrile (solvent B) was used throughout the analysis. Prior to the sample extract injection, the HPLC column was washed and conditioned with the mobile phase for $5 \mathrm{~min}$. Analysis was performed at $0.5 \mathrm{~mL} \mathrm{~min}{ }^{-1}$ flow rate, a column temperature of $30^{\circ} \mathrm{C}, 15 \mu \mathrm{L}$ injection volume and a monitoring wavelength of $224 \mathrm{~nm}$.

\subsection{Alcoholic Beverage Samples}

Three different kinds of alcoholic beverage samples were collected from local supermarkets in Addis Ababa, Ethiopia. Beer and red wine (Gouder red wine) samples which were manufactured by Meta Abo Brewery Share Company SC (Alem Gena, Sebeta, Ethiopia) and Awash Wine Share Company (Nefasilk Lafto sub-city, Addis Ababa, Ethiopia), respectively, were purchased from local market in Arada subcity of Addis Ababa. Ehtipian honey wine "Tej" was purchased from Mekanisa area, Nefasilk Lafto sub-city of Addis Ababa. The collected samples were stored in the dark at $4{ }^{\circ} \mathrm{C}$ until the analysis, without prior sample pretreatment.

\subsection{SALLE Procedure}

$2.5 \mathrm{~mL}$ alcoholic beverage sample was taken into a $15-\mathrm{mL}$ conical centrifuge tube. The sample was then spiked with appropriate concentrations of the target pesticides standards and kept for a minimum of $30 \mathrm{~min}$ to ensure homogeneity and attaining equilibration. Thereafter, $2.5 \mathrm{~mL}$ of $0.04 \mathrm{~mol} / \mathrm{L}$ phosphate buffer ( $\mathrm{pH} 7)$ and $1 \mathrm{~mL}$ acetonitrile were added, respectively and the content was then manually shaken for few seconds. Subsequently, $1.5 \mathrm{~g}$ of $\mathrm{MgSO}_{4}$ was added and the mixture was again shaken for a minute, before subjecting to centrifugation for $5 \mathrm{~min}$ at $4000 \mathrm{rpm}$ to enhance the phase separation. Eventually, $200 \mu \mathrm{L}$ of the upper layer organic phase was carefully withdrawn using a micropipette and transferred into insert vial, which was housed in a $1.5 \mathrm{~mL}$ 
autosampler vial and $15 \mu \mathrm{L}$ of the extract was then injected into HPLC system for separation and quantification.

\section{Results and Discussion}

\subsection{Optimization of SALLE Pprocedure}

The SALLE method, usually involves optimization of various experimental variables that are potentially affecting the extraction efficiency and selectivity of the method $[25$, $30,37]$. These variables include the type and concentration of salt, volume of acetonitrile (the most commonly used extraction solvent in SALLE procedure) and $\mathrm{pH}$ of the aqueous samples. In this study, optimization of the parameters was carried out using $5 \mathrm{~mL}$ deionized water containing $200 \mu \mathrm{g} \mathrm{L}^{-1}$ standards of the five target analytes. The average peak area of the replicate analyses was used to evaluate the extraction efficiency of different experimental parameters.

\subsubsection{Selection of Salt Type}

In SALLE procedure, the degree of phase separation can be varied with the types of salts used [30, 31, 37]. In this study, the effect of four salts including $\mathrm{NaCl}, \mathrm{MgSO}_{4}$, $\mathrm{Na}_{2} \mathrm{CO}_{3}$ and $\left(\mathrm{NH}_{4}\right)_{2} \mathrm{SO}_{4}$ each at $20 \%(\mathrm{~m} / \mathrm{V})$ concentration level, were tested as potential salting-out agents. With the exception $\mathrm{NaCl}$, the others have exhibited good phase separation. However, as can be seen from Figure 2, the highest peak areas of the analytes were observed when $\mathrm{MgSO}_{4}$ was used as salting-out agent. This could be attributed to the highest ionic strength per unit concentration of $\mathrm{MgSO}_{4}$ in aqueous solution as compared to the others [42]. Thus, $\mathrm{MgSO}_{4}$ was selected for subsequent experiments.

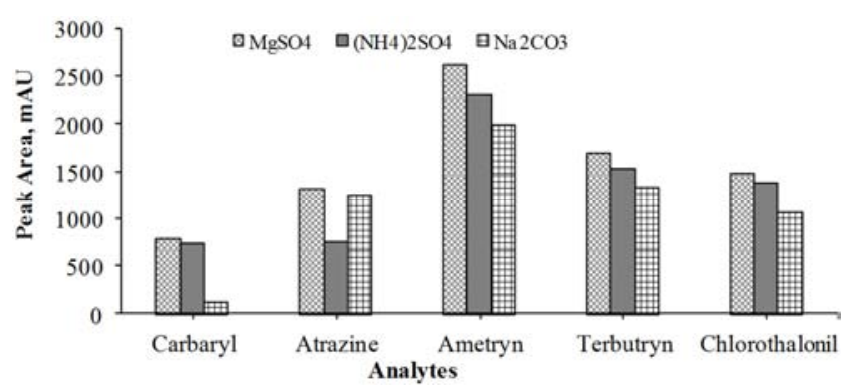

Figure 2. Effect of salt type on SALLE efficiency. Experimental conditions: extraction solvent volume, $1 \mathrm{~mL}$; centrifugation speed and time, $4000 \mathrm{rpm}$ and $5 \mathrm{~min}$, respectively.

\subsubsection{Effect of $\mathrm{MgSO}_{4}$ Concentration}

Concentration of the salt is also important factor affecting the extraction efficiency of the method [30, 37]. The concentration of the salt must be large enough to induce the required phase separation. Accordingly, different concentrations of $\mathrm{MgSO}_{4}$ ranging from $10-40 \%(\mathrm{~m} / \mathrm{v})$ in the sample solution were assessed. It was observed that peak areas of the target analytes increase with concentration of the salt up to $30 \%$ and then decline at higher concentration
(Figure 3). So, 30\%; $\mathrm{m} / \mathrm{v} \mathrm{MgSO} 4$ was chosen as the optimum concentration for the subsequent studies.

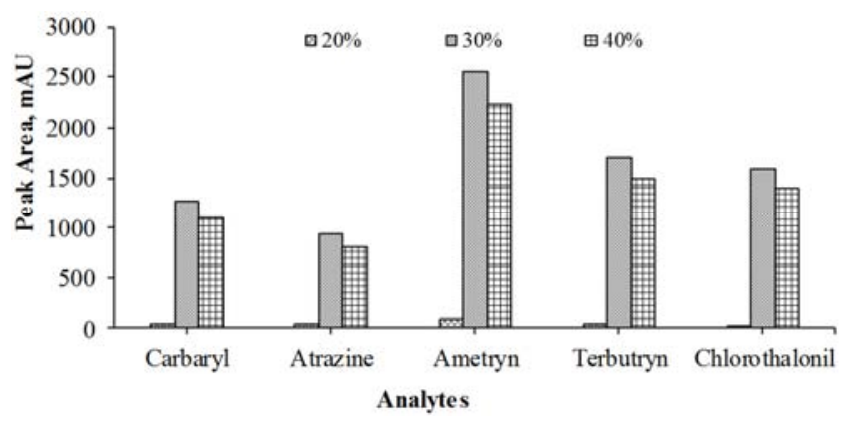

Figure 3. Effect of salt concentration on SALLE efficiency. Experimental conditions are mentioned in Figure 2.

\subsubsection{Effect of Acetonitrile Volume}

The volume of acetontrile, the extraction solvent, as well plays a great role on the extraction performance of the SALLE procedure [31, 37, 42]. The effect of the acetonitrile volume on the extraction performance was investigated by varying its volume over the range of $0.5-2.0 \mathrm{~mL}$. It was observed that the peak areas of the target analytes increased with the volume of the acetonirile from 0.5 to $1.0 \mathrm{~mL}$ and then decreased at higher volumes. At low volumes, phase separation between acetonitrile and aqueous phases was not clear and thus, collection of the organic phase was difficult. On the other hand, at higher volumes, the volume of organic phase obtained after phase separation is higher than its initial volume, indicating the existence of dissolved water in the organic phases [37, 42]. Based on the experimental results, 1 $\mathrm{mL}$ acetonitrile was selected as the optimum volume in the subsequent experiments.

\subsubsection{Effect of the Sample $\mathrm{pH}$}

For efficient extraction of ionizable and relatively polar compounds, $\mathrm{pH}$ of the sample solution plays a decisive role. The sample solution $\mathrm{pH}$ should be lower than the $\mathrm{pK}_{a}$ of the analytes to obtain the target analytes in their unionized forms so that they have higher tendency to partition into the organic phase $[9,43]$. A series of experiments were performed to investigate the effect of $\mathrm{pH}$ on the extraction efficiency of the SALLE method for the target analytes by adjusting the $\mathrm{pH}$ of the sample solution over the range of 4-8, keeping other experimental parameters constant. As shown in Figure 5, peak areas of all the target analytes increased with the rise in $\mathrm{pH}$ of the sample solution up to $\mathrm{pH} 7$ and then started to decrease on further increase in $\mathrm{pH}$ of the sample solution. The lower peak areas observed at higher $\mathrm{pH}$ might be most likely due to the hydrolysis of the pesticides [37]. On the other hand, the lower peak areas of the target analytes at lower $\mathrm{pH}$ might be attributed to their incomplete conversion to their neutral form and thus, complete transfer of the analytes from the sample solution to the organic phase could not be achieved. Therefore, a sample solution of $\mathrm{pH} 7$ was chosen as the optimum. 


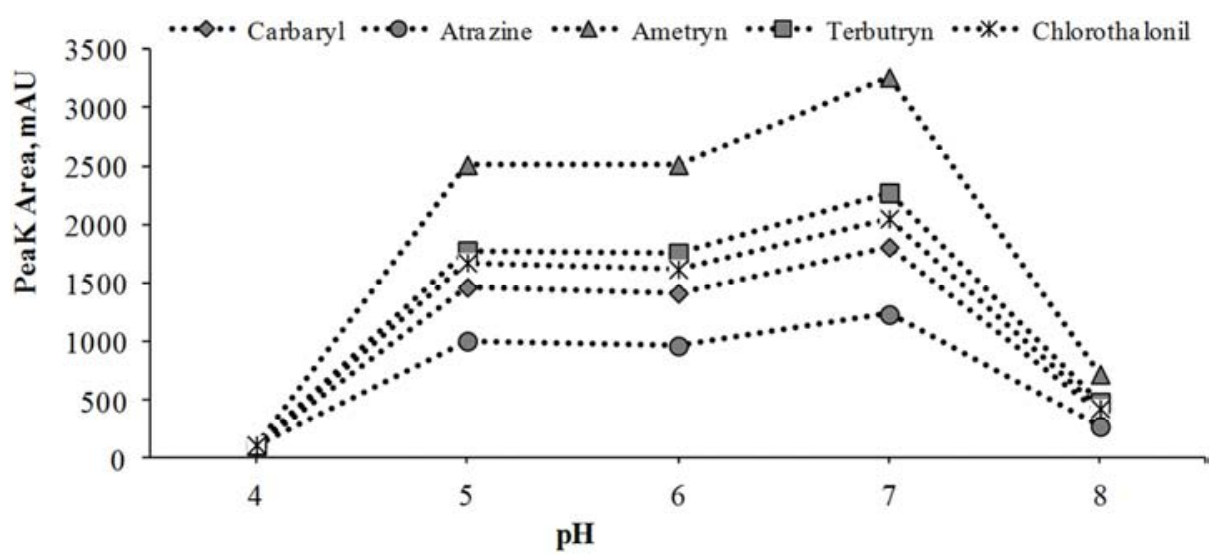

Figure 4. Effect of sample pH on SALLE efficiency. Experimental conditions: salt concentration, 30\% ( $\mathrm{m} / \mathrm{v})$; and others are mentioned in Figure 2.

\subsection{Validation of the Proposed Method}

\subsubsection{Calibration Curves and Analytical Performance Characteristics}

The proposed method was evaluated by constructing matrixmatched calibration curves using the beer sample as a representative matrix. The calibration curves were established by analyzing the extract of the spiked beer sample with the mixture of the analytes at six different concentration levels. Each level was extracted in duplicate and each extract was then injected in duplicate. Then, calibration curves were obtained by considering the average peak areas as the instrumental response versus the analytes concentrations. The coefficients of determination $\left(\mathrm{R}^{2}\right)$ for all the analytes were higher than 0.997 , indicating good linearity over the studied concentrations range. The limits of detection (LOD) and quantification (LOQ) were determined as the minimum analytes concentrations yielding 3 and 10 times the signal-tonoise $(\mathrm{S} / \mathrm{N})$ ratio, respectively. The performance characteristics of the proposed SALLE method are shown in Table 1.

\subsubsection{Precision Study}

The precision of the method was studied in terms of repeatability (intra-day) and intermediate (inter-day) precisions by extracting the spiked beer sample, at two concentration levels: Level 1: $50 \mu \mathrm{g} \mathrm{L}^{-1}$ and Level 2: $200 \mu \mathrm{g}$ $\mathrm{L}^{-1}$. Repeatability of the proposed method was investigated by extracting two spiked beer samples at both concentration levels and the obtained extracts were then injected in triplicate on the same day, under the same experimental conditions. Similarly, the intermediate precision of the method was also assayed by extracting one spiked beer sample at both concentration levels for three consecutive days. Each extract was then injected in triplicate. The results of both repeatability and intermediate precisions, which were expressed as the relative standard deviations (\%RSD) of the peak areas are shown in Table 2. Acceptable precisions, i.e., \%RSD less than 10.0, were obtained in all cases.

Table 1. Statistical and performance characteristics of the proposed SALLE method.

\begin{tabular}{lllll}
\hline Analytes & $\begin{array}{l}\text { Linear range } \\
\left(\boldsymbol{\mu g} \mathbf{~ L}^{-1}\right)\end{array}$ & $\mathbf{R}^{\mathbf{2}}$ & $\begin{array}{l}\mathbf{L O D} \\
\left(\boldsymbol{\mu g} \mathbf{~ L}^{-1}\right)\end{array}$ & $\begin{array}{l}\mathbf{L O Q} \\
\left(\boldsymbol{\mu g} \mathbf{~ L}^{-1}\right)\end{array}$ \\
\hline Carbaryl & $9-250$ & 0.998 & 2.6 & 8.6 \\
Atrazine & $11-250$ & 0.997 & 3.3 & 10.9 \\
Ametryn & $5-250$ & 0.997 & 1.3 & 4.5 \\
Terbutryn & $9-250$ & 0.997 & 2.8 & 9.3 \\
Chlorothal & $13-250$ & 0.999 & 3.9 & 12.8 \\
\hline
\end{tabular}

Table 2. Repeatability and intermediate precision of the proposed method (\%RSD) for the spiked beer samples.

\begin{tabular}{|c|c|c|c|c|}
\hline \multirow{2}{*}{ Analytes } & \multicolumn{2}{|c|}{ Repeatability $(n=6)$} & \multicolumn{2}{|c|}{ Intermediate precision $(\mathrm{n}=9)$} \\
\hline & Level 1 & Level 2 & Level 1 & Level 2 \\
\hline Carbaryl & 6.6 & 0.8 & 7.6 & 9.2 \\
\hline Atrazine & 6.2 & 1.3 & 9.3 & 8.7 \\
\hline Ametryn & 4.0 & 4.6 & 9.1 & 9.3 \\
\hline Terbutryn & 3.6 & 1.0 & 3.7 & 9.9 \\
\hline Chlorothalonil & 7.8 & 1.6 & 4.8 & 9.8 \\
\hline
\end{tabular}

\subsubsection{Applications and Recovery Studies}

The applicability of the developed method was investigated by performing recovery studies using three different types of alcoholic beverages including beer, wine and $T e j$ samples. For recovery studies, each sample was spiked at two concentration levels previously used for precision studies. Each concentration level was extracted in duplicate and each extract was injected in triplicate. In all cases, blank samples were extracted and analyzed by the proposed method; however, none of the target analytes was detected in these samples. Recoveries were calculated by comparing the concentration of the extracted analytes with the initial concentration of the target analytes spiked to the alcoholic beverage samples [44]. Recoveries and the corresponding \%RSD $(n=6)$ of each target analyte in beer, wine and Tej samples are shown in Table 3. The observed recoveries were in the range of $71-104 \%$ in all the samples. These results were in good agreement with the acceptable 
recovery range (i.e., from 70 to $120 \%$ ) established by the European Commission for pesticide residue analysis in food

and feed samples [45].

Table 3. Recovery values (\%) of the method for the spiked alcoholic beverages.

\begin{tabular}{lllllll}
\hline \multicolumn{2}{l}{ Recovery (\%RSD; $\boldsymbol{n}=\mathbf{6})$} & Carbaryl & Atrazine & Ametryn & Terbutryn & Chlorothalonil \\
\multirow{2}{*}{ Beer } & Level 1 & $104(1.3)$ & $71(1.6)$ & $80(1.5)$ & $89(6.5)$ & $87(2.1)$ \\
& Level 2 & $89(2.3)$ & $74(0.9)$ & $77(1.2)$ & $73(3.6)$ & $71(5.2)$ \\
\multirow{2}{*}{ Wine } & Level 1 & $101(4.9)$ & $90(5.7)$ & $78(4.3)$ & $88(5.8)$ & $79(7.8)$ \\
\multirow{2}{*}{$T e j$} & Level 2 & $86(4.0)$ & $72(2.6)$ & $74(4.1)$ & $71(3.8)$ & $72(4.1)$ \\
& Level 1 & $95(5.0)$ & $82(9.2)$ & $99(3.1)$ & $75(7.3)$ & $87(6.0)$ \\
& Level 2 & $91(5.9)$ & $83(8.9)$ & $93(2.5)$ & $87(3.0)$ & $76(4.0)$ \\
\hline
\end{tabular}

\subsubsection{Selectivity of the Analytical Technique}

Selectivity of the proposed method was evaluated by comparing the chromatograms of the unspiked alcoholic beverage samples with that of the corresponding spiked samples. Figure 5 shows representative chromatograms of the unspiked and spiked Tej samples with $50 \mu \mathrm{g} \mathrm{L}^{-1}$ of the target pesticides. As can be seen from the chromatograms, no interferences were observed at the retention time of the target analytes, indicating that the proposed method has good selectivity for trace level analysis of the selected pesticides in alcoholic beverages and other related matrices.

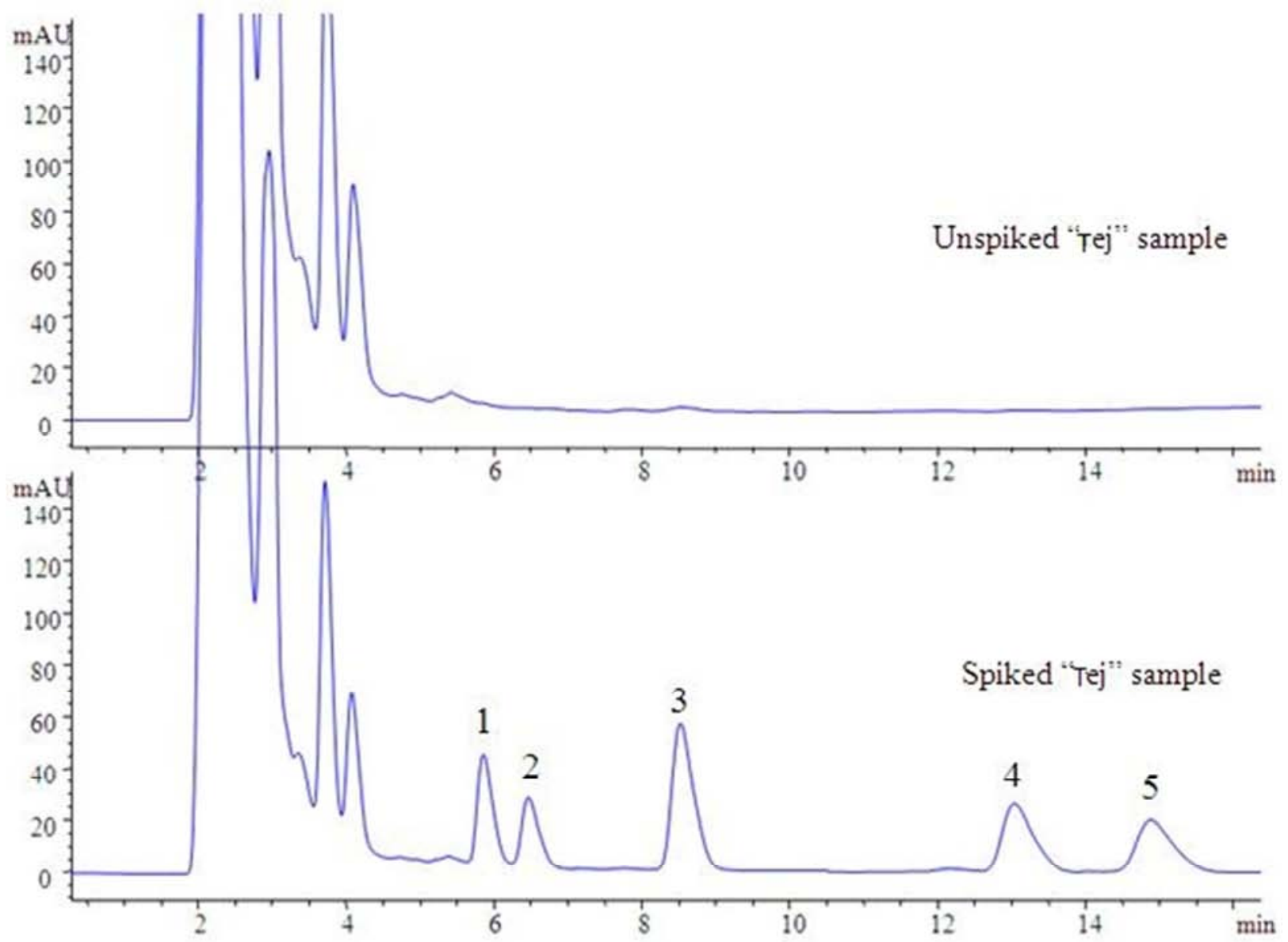

Figure 5. The chromatograms of unspiked and spiked Tej samples showing the selectivity of the proposed method $(1=$ Carbaryl, $2=$ Atrazine, $3=$ Ametryn, 4 $=$ Terbutryn, $5=$ Chlorothalonil).

\section{Conclusions}

In this study, a method that makes use of SALLE technique combined with HPLC-DAD has been proposed for determination of five multiclass pesticide residues in three different alcoholic beverages. Different parameters affecting the extraction performances of the target analytes such as the salt type and its concentration, acetonitrile (extraction solvent) volume and $\mathrm{pH}$ were investigated and the optimum conditions were established. Using the optimum conditions, the proposed method exhibited its usefulness for the analysis of the target analytes, with good LODs and LOQs, good selectivity, acceptable precisions, wide linearity ranges and satisfactory recoveries. The method has advantages of simplicity, easy operation and short analysis time with 
consumption of low volume of the less hazardous organic solvent, acetonitrile. Therefore, the developed method can be utilized as an attractive alternative for the determination of multiclass pesticides residues in various alcoholic beverages and other matrices.

\section{Acknowledgements}

Authors are grateful to the Department of Chemistry of the Addis Ababa University (AAU) for providing the laboratory facilities. Financial support for purchase of the analytical pesticide standards, HPLC grade solvents and other necessary expenses both for practical works as well as field studies were obtained from International Science Program (ISP) through "Trace Level Pollutants Analysis" project; ETH:04.

\section{References}

[1] F. E. Ahmed. (2001). Analyses of Pesticides and Their Metabolites in Foods and Drinks. Trends Anal. Chem. 20: 649-66.

[2] M. Lee, M. Regu and S. Seleshe. (2015). Uniqueness of Ethiopian Traditional Alcoholic Beverage of Plant Origin. tella. J. Ethn. Foods 2: 110-114.

[3] A. Gure, F. J. Lara, A. M. García-Campaña, N. Megersa and M. del Olmo-Iruela. (2015). Vortex-assisted Ionic Liquid Dispersive Liquid-Liquid Microextraction for the Determination of Sulfonylurea Herbicides in Wine Samples By Capillary High-Performance Liquid Chromatography. Food Chem. 170: 348-353.

[4] P. Plaza Bolanos, R. Romero-González, A. Garrido Frenich and J. L. Martínez Vidal. (2008). Application of Hollow-fibre Liquid Phase Microextraction for the Multiresidue Determination of Pesticides in Alcoholic Beverages by UltraHigh Pressure Liquid Chromatography Coupled to Tandem Mass Spectrometry. J. Chromatogr. A 1208: 16-24.

[5] J. Martins, C. Esteves, A. Limpo-Faria, P. Barros, N. Ribeiro, T. Simoes, M. Correia and C. Delerue-Matos. (2012). Analysis of Six Fungicides and one Acaricide in Still and Fortified Wines Using Solid-Phase Microextraction-Gas Chromatography/Tandem Mass Spectrometry. Food Chem. 132: 630-636.

[6] P. Jovanov, V. Guzsvány, M. Franko, S. Lazić, M. Sakač, I. Milovanović and N. Nedeljković. (2014). Development of Multiresidue DLLME and QuEChERS Based LC-MS/MS Method for Determination of Selected Neonicotinoid Insecticides in Honey Liqueur. Food Res. Int. 55: 11-19.

[7] J. W. Wong, M. G. Webster, C. A. Halverson, M. J. Hengel, K. K. Ngim and S. E. Ebeler. (2003). Multiresidue Pesticide Analysis in Wines by Solid-Phase Extraction and Capillary Gas Chromatography-Mass Spectrometric Detection with Selective Ion Monitoring. J. Agric. Food chem. 5: 1148-1161.

[8] J. W. Wong, M. G. Webster, D. Z. Bezabeh, M. J. Hengel, K. K. Ngim, A. J. Krynitsky and S. E. Ebeler. (2004). Multiresidue Determination of Pesticides in Malt Beverages by Capillary Gas Chromatography with Mass Spectrometry and Selected Ion Monitoring. J. Agric. Food Chem. 52: 6361-
6372.

[9] N. Megersa and S. Kassahun. (2012). A New Selective Liquid Membrane Extraction Method for the Determination of Basic Herbicides in Agro-Processed Fruit Juices and Ethiopian Honey Wine (Tej) Samples. Food Addit. Contam. 29: 789 798.

[10] M. A. Gonzalez-Curbelo, A. V. Herrera-Herrera, L. M. Ravelo-Perez and J. Hernandez-Borges. (2012). SamplePreparation Methods for Pesticide-Residue Analysis in Cereals and Derivatives. Trends Anal. Chem. 38: 32-51.

[11] Regulation (EC) No. 396/2005 of the European Parliament and of the Council on Maximum Residue Levels of Pesticides in or on Food and Feed of Plant and Animal Origin and Amending Council Directive 91/414/EEC. Official Journal of the European Communities. No. L 70, 16 March 2005, pp. 116.

[12] Regulation (EC) No. 149/2008 Amending Regulation (EC) No. 396/2005 of the European Parliament and of The Council by Establishing Annexes II, III and IV Setting Maximum Residue Levels for Products Covered by Annex I Thereto. Official Journal of the European Communities. No. L 58, 1 March 2008, pp. 1-398.

[13] T. Hyötyläinen, T. Tuutijärvi, K. Kuosmanen and M. L. Riekkola. (2002). Determination of Pesticide Residues in Red Wines with Microporous Membrane Liquid-Liquid Extraction and Gas Chromatography. Anal. Bioanal. Chem. 372: 732736 .

[14] T. Hyötyläinen, K. Lüthje, M. Rautiainen-Rämä and M. L. Riekkola. (2004). Determination of pesticides in red wines with on-line coupled microporous membrane liquid-liquid extraction-gas chromatography. J. Chromatogr. A 1056: $267-$ 271.

[15] J. J. Jimenéz, J. L. Bernal, M. J. del Nozal, L. Toribio and E. Arias. (2001). Analysis of Pesticide Residues in Wine by Solid-Phase Extraction and Gas Chromatography with Electron Capture and Nitrogen-Phosphorus Detection. $J$. Chromatogr. A 919: 147-156.

[16] G. Cinelli, P. Avino, I. Notardonato and M. V. Russo. (2014). Ultrasound-Vortex-Assisted Dispersive Liquid-Liquid Microextraction Coupled with Gas Chromatography with a Nitrogen-Phosphorus Detector for Simultaneous and Rapid Determination of Organophosphorus Pesticides and Triazines in Wine. Anal. Methods 6: 782-790.

[17] N. Chen, H. Gao, N. Ye, Q. Zhong, Z. Xiong and X. Gu. (2012). Fast Determination of 22 Pesticides in Rice Wine by Dispersive Solid-Phase Extraction in Combination with GCMS. Am. J. Anal. Chem. 3: 33-39.

[18] A. Economou, H. Botitsi, S. Antoniou and D. Tsipi. (2009). Determination of Multi-Class Pesticides in Wines by SolidPhase Extraction and Liquid Chromatography-Tandem Mass Spectrometry. J. Chromatogr. A 1216: 5856-5867.

[19] N. Vela, G. Pérez, G. Navarro and S. Navarro. (2007). Gas Chromatographic Determination of Pesticide Residues in Malt, Spent Grains, Wort and Beer with Electron Capture Detection and Mass Spectrometry. J. AOAC Int. 90: 544-549.

[20] M. J. Hengel, D. Miller and R. Jordan. (2016). Development and Validation of a Method for the Determination of Pesticide Residues in Beer by Liquid Chromatography-Mass Spectrometry. J. Am. Soc. Brew. Chem. 74: 49-52. 
[21] M. Ashenafi. (2006). A review on the Microbiology of Indigenous Fermented Foods and Beverages of Ethiopia. Ethiop. J. Biol. Sci, 5: 189-245.

[22] T. Yohannes, F. Melak and K. Siraj. (2013). Preparation and Physicochemical Analysis of Some Ethiopian Traditional Alcoholic Beverages. Afr. J. Food Sci. 7: 399-403.

[23] P. Helena and Z. K. Locija. (1999). Solid-Phase Microextraction. Trends Anal. Chem. 18: 272-282.

[24] I. M. Valente, L. M. Goncalves and J. A. Rodrigues. (2013). Another Glimpse Over the Salting-Out Assisted Liquid-Liquid Extraction in Acetonitrile/Water Mixtures. J. Chromatogr. A 1308: 58-62.

[25] I. M. Valente and J. A. Rodrigues. (2016). Determination of $\alpha-$ Dicarbonyls in Wines Using Salting-Out Assisted LiquidLiquid Extraction. LCGC 34: 32-37.

[26] A. Jain, M. Gupta and K. K. Verma. (2015). Salting-out Assisted Liquid-Liquid Extraction for the Determination of Biogenic Amines in Fruit Juices and Alcoholic Beverages after Derivatization with 1-Naphthylisothiocyanate and High Performance Liquid Chromatography. J. Chromatogr. A 1422: 60-72.

[27] H. Dong, X. Guo, Y. Xian, H. Luo, B. Wang and Y. Wu. (2015). A salting out-Acetonitrile Homogeneous Extraction Coupled with Gas Chromatography-Mass Spectrometry Method for The Simultaneous Determination of Thirteen NNitrosamines in Skin Care Cosmetics. J. Chromatogr. A 1422: 82-88.

[28] X. Xiong and L. Yang. (2015). Salting-out-Assisted LiquidLiquid Extraction with Acetonitrile for the Determination of Trimetazidine in Rat Plasma Using Liquid ChromatographyMass Spectrometry. Biomed. Chromatogr. 29: 268-274.

[29] K. Hajkova, B. Jurasek, D. Sykora, T. Palenicek, P. Miksatkova and M. Kuchar. (2016). Salting-out-Assisted Liquid-Liquid Extraction as a Suitable Approach for Determination of Methoxetamine in Large Sets of Tissue Samples. Anal. Bioanal. Chem. 408: 1171-1181.

[30] J. Liu, M. Jiang, G. Li, L. Xu and M. Xie. (2010). Miniaturized Salting-out Liquid-Liquid Extraction of Sulfonamides from Different Matrices. Anal. Chim. Acta 679: $74-80$.

[31] H. Sereshti, M. Khosraviani and M. S. Amini-Fazl. (2014). Miniaturized salting-out Liquid-Liquid Extraction in a Coupled-Syringe System Combined with HPLC-UV For extraction and Determination of Sulfanilamide. Talanta 121: 199-204.

[32] D. Jain, R. Athawale, A. Bajaj, and S. Shrikhande (2014). Double-salting out Assisted Liquid-Liquid Extraction (SALLE) HPLC Method for Estimation of Temozolomide from Biological Samples. J. Chromatogr. B 970: 86-94.

[33] S. Ahmed and A. M. Mahmoud. (2015). A Novel Salting-out Assisted Extraction Coupled with HPLC- Fluorescence Detection for Trace Determination of Vitamin K Homologues in Human Plasma. Talanta 144: 480-487.

[34] S. Song, E. N. Ediage, A. Wu and S. De Saeger. (2013). Development and Application of Salting-out Assisted Liquid/Liquid Extraction for Multi-Mycotoxin Biomarkers Analysis in Pig Urine with High Performance Liquid Chromatography/Tandem Mass Spectrometry. J. Chromatogr.
A 1292: 111-120.

[35] D. Moreno-González, R. Rodríguez-Ramírez, M. del OlmoIruela and A. M. García-Campaña. (2017). Validation of a New Method Based on Salting-out Assisted Liquid-Liquid Extraction and UHPLC-MS/MS for the Determination of Betalactam Antibiotics in Infant Dairy Products. Talanta. 167: 493-498.

[36] Y. Wen, J. Li, F. Yang, W. Zhang, W. Liao, C. Li and L. Chen. (2013). Salting-out Assisted Liquid-Liquid Extraction with the Aid of Experimental Design for Determination of Benzimidazole Fungicides in High Salinity Samples by HighPerformance Liquid Chromatography. Talanta 106: 119-126.

[37] A. Gure, F. J. Lara, D. Moreno-González, N. Megersa, M. del Olmo-Iruela and A. M. García-Campaña. (2014). Salting-out Assisted Liquid-Liquid Extraction Combined with Capillary HPLC for The Determination of Sulfonylurea Herbicides Iin Environmental Water and Banana Juice Samples. Talanta 127: $51-58$.

[38] X. Y Xu, J. Ye, J. Nie, Z. G. Li and M. R. Lee. (2015). New Liquid-Liquid Microextraction Method by Ultrasound Assisted Salting-out for Determination of Triazole Pesticides in Water Samples Coupled by Gas Chromatography-Mass Spectrometry. Anal. Methods 7: 1194-1199.

[39] S. C. Natina, L. G. Kaldon and D. L. Bailey. (2015). Ammonium Salting-out Extraction with Analyte Preconcentration for Sub-Part per Billion Quantitative Analysis in Surface, Ground and Drinking Water by Flow Injection Tandem Mass Spectrometry. Anal. Methods 7: 23002312 .

[40] Y. Fan, S. Hu and S. Liu. (2014). Salting-out Assisted LiquidLiquid Extraction Coupled to Dispersive Liquid-Liquid Microextraction for The Determination of Chlorophenols in Wine by High-Performance Liquid Chromatography. J. Sep. Sci. 37: 3662-3668.

[41] T. Bedassa, A. Gure and N. Megersa. (2015). Modified QuEChERS Method for the Determination of Multiclass Pesticide Residues in Fruit Samples Utilizing HighPerformance Liquid Chromatography. Food Anal. Methods 8: 2020-2027.

[42] J. Zhang, H. Wu, E. Kim and T. A. El-Shourbagy. (2009). Salting-out Assisted Liquid/Liquid Extraction with Acetonitrile: A New High Throughput Sample Preparation Technique for Good Laboratory Practice Bioanalysis Using Liquid Chromatography-Mass Spectrometry. Biomed. Chromatogr. 23: 419-425.

[43] A. Tapparo, D. Marton, C. Giorio, A. Zanella, L. Soldà, M. Marzaro, V. Linda and G. Vincenzo. (2012). Assessment of the Environmental Exposure of Honeybees to Particulate Matter Containing Neonicotinoid Insecticides Coming from Corn Coated Seeds. Environ. Sci. Technol. 46: 2592-2599.

[44] R. P. Carneiro, F. A. S Oliveira, F. D. Madureira, G. Silva, W. R. de Souza and R. P. Lopes. (2013). Development and Method Validation for Determination of 128 Pesticides in Bananas by Modified QuEChERS and UHPLC-MS/MS Analysis. Food Cont. 33: 413-423.

[45] Guidance Document on Analytical Quality Control and Validation Procedures for Pesticide Residues Analysis in Food and Feed. European Commission SANTE/11945/2015 Supersedes SANCO/12571/2013, Implemented by 01/01/2016 